TRANSACTIONS OF THE

AMERICAN MATHEMATICAL SOCIETY

Volume 335, Number 1, January 1993

\title{
A DYNAMICAL PROOF OF THE MULTIPLICATIVE ERGODIC THEOREM
}

\author{
PETER WALTERS
}

\begin{abstract}
We shall give a proof of the following result of Oseledec, in which $G L(d)$ denotes the space of invertible $d \times d$ real matrices, $\|\cdot\|$ denotes any norm on the space of $d \times d$ matrices, and $\log ^{+}(t)=\max (0, \log (t))$ for $t \in[0, \infty)$.
\end{abstract}

Oseledec's Multiplicative Ergodic Theorem [7]. Let $T$ be a measure-preserving transformation of a Lebesgue probability space $(X, \mathscr{B}, m)$ and let $x \rightarrow A_{x}$ be a measurable map of $X$ into $G L(d)$ such that the real-valued functions $x \rightarrow$ $\log ^{+}\left\|A_{x}\right\|, x \rightarrow \log ^{+}\left\|\left(A_{x}\right)^{-1}\right\|$ are integrable. There exists $Y \in \mathscr{B}$ with $T Y \subset$ $Y$ and $m(Y)=1$ such that

(i) there is a measurable function $s: Y \rightarrow \mathbb{N}$ with $s \circ T=s$;

(ii) for each $x \in Y$ there are real numbers $\lambda^{(s(x))}(x)<\lambda^{(s(x)-1)}(x)<\cdots<$ $\lambda^{(2)}(x)<\lambda^{(1)}(x)$ with $\lambda^{(i)}(T x)=\lambda^{(i)}(x)$ when $1 \leq i \leq s(x)$, and $x \rightarrow \lambda^{(i)}(x)$ is measurable on $\{x \in Y \mid s(x) \geq i\}$;

(iii) for each $x \in Y$ there are linear subspaces,

$$
\{0\} \equiv V_{x}^{(s(x)+1)} \subset V_{x}^{(s(x))} \subset \cdots \subset V_{x}^{(2)} \subset V_{x}^{(1)}=R^{d},
$$

of $R^{d}$ with $A_{x} V_{x}^{(i)}=V_{T x}^{(i)}$, and $x \rightarrow V_{x}^{(i)}$ is a measurable map from $\{x \in Y \mid s(x) \geq i\}$ into the Grassmannian of $R^{d}$;

(iv) $\forall x \in Y \forall v \in V_{x}^{(i)} \backslash V_{x}^{(i+1)}, \frac{1}{n} \log \left\|A_{T^{n-1} x} \cdots A_{T x} \cdot A_{x} v\right\| \rightarrow \lambda^{(i)}(x)$ where $\|\cdot\|$ denotes any norm on $R^{d}$.

The proof we give is a dynamical proof free of most of the matrix calculations of previous proofs $[7,9,10,6,3]$. As indicated in [8] it is quite straightforward to obtain (i), (ii), (iii) above and to get (iv) with the limit replaced by limit superior (see $\S 1$ ). The work comes in showing the limit exists in (iv) and this is done by using two results, Theorems 11 and 13, the second of which considers the ergodic theory of a transformation on $X \times P\left(R^{d}\right)$ where $P\left(R^{d}\right)$ is the projective space obtained from $R^{d}$. We have only been able to use the second result under the assumptions that both $x \rightarrow \log ^{+}\left\|A_{x}\right\|, x \rightarrow \log ^{+}\left\|\left(A_{x}\right)^{-1}\right\|$ are integrable (which is equivalent to requiring $x \rightarrow \log \left\|A_{x}\right\|, x \rightarrow \log \left\|\left(A_{x}\right)^{-1}\right\|$ are integrable), whereas Oseledec's theorem is true under only the first assumption but in this case $\lambda^{(s(x))}(x)$ can be $-\infty$. We have indicated where the second

Received by the editors February 7, 1990 and, in revised form, October 5, 1990.

1980 Mathematics Subject Classification (1985 Revision). Primary 28D05; Secondary 58F11.

Key words and phrases. Ergodic theorem, measure-preserving, ergodic. 
assumption enters the proof. The numbers $\lambda^{(i)}(x)$ are called the Lyapunov characteristic exponents of $(T, A)$ at $x$.

We shall use $\mathscr{M}(d)$ to denote the space of all real $d \times d$ matrices and $\|\cdot\|$ to denote any norm on this vector space. The natural basis in $R^{d}$ is denoted by $\left\{e_{1}, e_{2}, \ldots, e_{d}\right\}$, and we always consider the Euclidean inner-product on $R^{d}$. From now on $(X, \mathscr{B}, m)$ will be a Lebesgue probability space and $T: X \rightarrow X$ a measure-preserving transformation. Hence $m$ is a complete measure and the ergodic decomposition holds. Also $A: X \rightarrow \mathscr{M}(d)\left(x \rightarrow A_{x}\right)$ will be a measurable map with $x \rightarrow \log ^{+}\left\|A_{x}\right\|$ integrable. For $n>0,\left(A^{n}\right)_{x}$ denotes $A_{T^{n-1} x} \cdots A_{T x} . A_{x}$ (matrix multiplication).

If $P$ is a compact metric space equipped with its Borel $\sigma$-algebra $\mathscr{B}(P)$, then $M_{m}(X \times P)$ denotes the space of probability measures on $X \times P$ that project to $m$ on $X$, and if $S: X \times P \rightarrow X \times P$ is a measurable map covering $T$ then $M_{m}(X \times P, S)$ denotes those members of $M_{m}(X \times P)$ which are invariant under $S$.

\section{Proof of (i), (ii), (iii) AND MOST of (iv)}

We shall use the following simple result about sequences of real numbers.

Lemma 1. If $a_{n}, b_{n} \geq 0$ for $n \geq 1$ then

$$
\limsup _{n \rightarrow+\infty} \frac{1}{n} \log \left(a_{n}+b_{n}\right)=\max \left(\limsup _{n \rightarrow+\infty} \frac{1}{n} \log a_{n}, \limsup _{n \rightarrow+\infty} \frac{1}{n} \log b_{n}\right)
$$

and

$$
\liminf _{n \rightarrow+\infty} \frac{1}{n} \log \left(a_{n}+b_{n}\right) \geq \max \left(\liminf _{n \rightarrow+\infty} \frac{1}{n} \log a_{n}, \liminf _{n \rightarrow+\infty} \frac{1}{n} \log b_{n}\right) .
$$

Lemma 2. Suppose $T: X \rightarrow X$ is a measure-preserving transformation of ( $X$, $\mathscr{B}, m)$ and let $A: X \rightarrow \mathscr{M}(d)$ be such that $x \rightarrow \log ^{+}\left\|A_{x}\right\|$ is integrable. Define $\chi: X \times R^{d} \rightarrow R \cup\{ \pm \infty\}$ by $\chi(x, v)=\lim \sup _{n \rightarrow+\infty} \frac{1}{n} \log \left\|\left(A^{n}\right)_{x} v\right\|$. Then

(i) there exists $X_{1} \in \mathscr{B}$ with $m\left(X_{1}\right)=1$ and $T X_{1} \subset X_{1}$ such that $\chi(x, v) \in R \cup\{-\infty\} \forall x \in X_{1}, \forall v \in R^{d}$

(ii) $\chi(x, 0)=-\infty \forall x \in X$;

(iii) $\chi(x, v)=\chi(x, a v) \forall a \in R \backslash\{0\} \forall v \in R^{d} \forall x \in X$;

(iv) $\chi\left(x, v_{1}+v_{2}\right) \leq \max \left(\chi\left(x, v_{1}\right), \chi\left(x, v_{2}\right)\right) \forall v_{1}, v_{2} \in R^{d} \forall x \in X$;

(v) $\chi\left(T x, A_{x} v\right)=\chi(x, v) \forall v \in R^{d} \forall x \in X$.

Proof. (i) $\left\|\left(A^{n}\right)_{x} v\right\| \leq\left\|\left(A^{n}\right)_{x}\right\| \cdot\|v\| \leq\left(\prod_{i=0}^{n-1}\left\|A_{T^{i} x}\right\|\right)\|v\|$ and since $x \rightarrow$ $\log ^{+}\left\|A_{x}\right\|$ is integrable $\chi(x, v) \in R \cup\{-\infty\}$ by Birkhoff's ergodic theorem.

The proofs of (ii) and (iii) are clear and (iv) follows from Lemma 1. Part (v) follows since

$$
\begin{aligned}
\chi\left(T x, A_{x} v\right) & =\limsup _{n \rightarrow+\infty} \frac{1}{n} \log \left\|\left(A^{n+1}\right)_{x} v\right\| \\
& =\limsup _{n \rightarrow+\infty} \frac{n+1}{n} \cdot \frac{1}{n+1} \log \left\|\left(A^{n+1}\right)_{x} v\right\| \\
& =\chi(x, v) .
\end{aligned}
$$


Corollary 3. With the notation of Lemma 2 we have

(i) $\forall x \in X_{1} \forall t \in R$ the set $V_{x}(t)=\left\{v \in R^{d} \mid \chi(x, v) \leq t\right\}$ is a linear subspace of $R^{d}$. We have $A_{x} V_{x}(t) \subset V_{T x}(t)$. Also $s \leq t$ implies $V_{x}(s) \subset$ $V_{x}(t)$.

(ii) $\forall x \in X_{1}, \chi(x,):. R^{d} \rightarrow R \cup\{-\infty\}$ takes only a finite number, $s(x)$, of different values $\lambda^{(s(x))}(x)<\lambda^{(s(x)-1)}(x)<\cdots<\lambda^{(1)}(x)$, where $\lambda^{(s(x))}(x)$ could be $-\infty$. We have $s(T x) \geq s(x)$, and $\lambda^{(s(x))}(x), \ldots, \lambda^{(1)}(x)$ are among the values $\left\{\lambda^{(j)}(T x): 1 \leq j \leq s(T x)\right\}$.

(iii) If, for $x \in X_{1}$, we define $V_{x}^{(i)}$ to be $V_{x}\left(\lambda^{(i)}(x)\right), 1 \leq i \leq s(x)$, then

$$
\{0\} \equiv V_{x}^{(s(x)+1)} \subset V_{x}^{(s(x))} \subset \cdots \subset V_{x}^{(2)} \subset V_{x}^{(1)}=R^{d}
$$

and

$v \in V_{x}^{(i)} \backslash V_{x}^{(i+1)} \Leftrightarrow \limsup _{n \rightarrow+\infty} \frac{1}{n} \log \left\|\left(A^{n}\right)_{x} v\right\|=\lambda^{(i)}(x), \quad 1 \leq i \leq s(x)$.

Proof.

(i) Each $V_{x}(t)$ is a linear subspace of $R^{d}$ by Lemma 2(iii) and (iv). Part (v) of Lemma 2 gives $A_{x} V_{x}(t) \subset V_{T x}(t)$. The last claim is clear.

(ii) Fix $x \in X_{1}$. Since $s<t$ implies $V_{x}(s) \subset V_{x}(t)$ and hence $\operatorname{dim}\left(V_{x}(s)\right) \leq$ $\operatorname{dim}\left(V_{x}(t)\right)$, we can let $\lambda^{(s(x))}<\cdots<\lambda^{(1)}(x)$ be the values of $t$ where $t \rightarrow \operatorname{dim}\left(V_{x}(t)\right)$ changes. Therefore $V_{x}\left(\lambda^{(i)}(x)\right)=\left\{v \in R^{d} \mid \chi(x, v) \leq\right.$ $\left.\lambda^{(i)}(x)\right\}$, and $\chi(x,$.$) can only take the values \lambda^{(s(x))}(x), \ldots, \lambda^{(1)}(x)$. The last statement follows from Lemma 2(v).

(iii) This part is now clear from the proof of (ii).

Lemma 4. With the notation of Lemma 2,

(i) $s: X_{1} \rightarrow \mathbb{N}$ is measurable and hence $\exists X_{2} \in \mathscr{B}$ with $m\left(X_{2}\right)=1, T X_{2} \subset$ $X_{2}$, and $s \circ T=s$ on $X_{2}$;

(ii) $\lambda^{(i)}:\left\{x \in X_{1} \mid s(x) \geq i\right\} \rightarrow R \cup\{-\infty\}$ is measurable and $\lambda^{(i)}(T x)=$ $\lambda^{(i)}(x) \forall x \in X_{2} \cap\{x \mid s(x) \geq i\}$

(iii) $x \rightarrow V_{x}^{(i)}$ is a measurable map from $\left\{x \in X_{1} \mid s(x) \geq i\right\}$ into the Grassmannian of $R^{d}$ and $A_{x} V_{x}^{(i)} \subset V_{T x}^{(i)}$.

Proof. We shall deal with the measurability questions in the next section. From Corollary 3(ii) we have $s \circ T \geq s$ so $s \circ T=s$ a.e. We get $\lambda^{(i)} \circ T=\lambda^{(i)}$ a.e. by Corollary 3 (ii).

To prove Oseledec's theorem we need to show that for a.e. $x \in X$ $\lim _{n \rightarrow \infty} \frac{1}{n} \log \left\|\left(A^{n}\right)_{x} v\right\|$ exists $\forall v \in R^{d}$. The rest of the paper, after $\S 2$, is devoted to this.

\section{Measurability Questions}

If $Y$ is a complete separable metric space let $\mathscr{P}_{K}(Y)$ denote the collection of all nonempty compact subsets of $Y$. We can equip $\mathscr{P}_{K}(Y)$ with the Hausdorff metric and it becomes a complete separable metric space. The following result is from [2, pp. 80 and 62]. 
Theorem 5 [2]. Let $(X, \mathscr{B}, m)$ be a complete probability space and let $Y$ be a complete separable metric space. Let $\Gamma: X \rightarrow \mathscr{P}_{K}(Y)$ be a map. The following statements are equivalent:

(a) $\Gamma$ is measurable (using the Borel $\sigma$-algebra on the metric space $\mathscr{P}_{K}(Y)$ ).

(b) $\forall$ open subset $U$ of $Y,\{x \in X \mid \Gamma(x) \cap U \neq \varnothing\} \in \mathscr{B}$.

(c) There is a sequence $\left\{\sigma_{n}\right\}$ of measurable maps $\sigma_{n}: X \rightarrow Y$ with $\sigma_{n}(x) \in$ $\Gamma(x) \forall x \in X$ and $\Gamma(x)=\left\{\sigma_{n}(x)\right\}_{n=1}^{\infty}$.

(d) $\operatorname{graph}(\Gamma) \equiv\{(x, y) \mid y \in \Gamma(x)\} \in \mathscr{B} \times \mathscr{B}(Y)$.

We also have

Theorem 6 [2, p. 75]. If $(X, \mathscr{B}, m)$ is a complete probability space and $Y$ is a complete separable metric space consider the natural projection from $X \times Y$ to $X$. If $D \in \mathscr{B} \times \mathscr{B}(Y)$ the projection of $D$ to $X$ is in $\mathscr{B}$.

If $V$ is a real vector space we let $P(V)$ denote the corresponding projective space obtained by collapsing each line through the origin of $V$ to a point. The topology on $P\left(R^{d}\right)$ is the quotient topology. Let $G\left(R^{d}\right)$ denote the Grassmannian of $R^{d}$, which consists of all linear subspaces of $R^{d}$ suitably topologized, and let $G_{k}\left(R^{d}\right)$ denote the space of all $k$-dimensional linear subspaces of $R^{d}$ [5]. We have

Theorem 7. Let $(X, \mathscr{B}, m)$ be a complete probability space and let a map $x \rightarrow V_{x}$ of $X$ into $G\left(R^{d}\right)$ be given. Define $r: X \rightarrow \mathbb{N}$ by $r(x)=\operatorname{dim} V_{x}$. The following are equivalent.

1. $x \rightarrow V_{x}$ is a measurable map of $X$ into $G\left(R^{d}\right)$.

2. $\left\{(x, v) \mid x \in X, v \in V_{x}\right\} \in \mathscr{B} \times \mathscr{B}\left(R^{d}\right)$.

3. $\left\{(x, y) \mid x \in X, y \in P\left(V_{x}\right)\right\} \in \mathscr{B} \times \mathscr{B}\left(P\left(R^{d}\right)\right)$.

4. $x \rightarrow P\left(V_{x}\right)$ is a measurable map of $X$ into $\mathscr{P}_{K}\left(P\left(R^{d}\right)\right)$.

5. $r: X \rightarrow R$ is measurable and for each $k, x \rightarrow V_{x}$ is a measurable map of $r^{-1}(k)$ into $G_{k}\left(R^{d}\right)$.

6. $r: X \rightarrow R$ is measurable and for each $k$ there are measurable maps $v_{1}, \ldots, v_{k}: r^{-1}(k) \rightarrow R^{d}$ such that $\forall x \in r^{-1}(k),\left\{v_{1}(x), \ldots, v_{k}(x)\right\}$ is an orthonormal basis for $V_{x}$.

7. $r: X \rightarrow R$ is measurable and for each $k$ there is a bimeasurable bijection from $\left\{(x, v) \mid x \in r^{-1}(k), v \in V_{x}\right\}$ onto $r^{-1}(k) \times R^{k}$ which is linear on fibres and covers the identity map of $X$.

8. $r: X \rightarrow R$ is measurable and for each $k$ there are measurable maps $u_{1}, \ldots, u_{k}: r^{-1}(k) \rightarrow R^{d}$ such that $\forall x \in r^{-1}(k),\left\{u_{1}(x), \ldots, u_{k}(x)\right\}$ is a basis for $V_{x}$.

Proof. Clearly (6) and (8) are equivalent by using the Gram-Schmidt process. Also (7) and (8) are clearly equivalent. Since the map $E \rightarrow P(E)$ of $G\left(R^{d}\right)$ into $\mathscr{P}_{K}\left(P\left(R^{d}\right)\right)$ is injective and continuous it is a homeomorphism onto its image and hence (1) is equivalent to (4). By Theorem 5, (3) and (4) are equivalent. Clearly (3) implies (2). To show that (2) implies (3) it suffices to show, by Theorem 5, that for each open subset $U$ of $P\left(R^{d}\right)$ we have $\left\{x \in X \mid P\left(V_{x}\right) \cap U \neq \varnothing\right\} \in \mathscr{B}$. If $q: X \times R^{d} \rightarrow X$ denotes projection to the first factor and $\pi: R^{d} \rightarrow P\left(R^{d}\right)$ denotes the natural projection then $\left\{x \in X \mid P\left(V_{x}\right) \cap U \neq \varnothing\right\}=q\left(\left\{(x, v) \mid x \in X, v \in V_{x}\right\} \cap\left(X \times \pi^{-1} U\right)\right)$ which 
belongs to $\mathscr{B}$ by Theorem 6 . Therefore (2) and (3) are equivalent. Since the dimension function is continuous on $G\left(R^{d}\right)$ we get (1) is equivalent to (5).

It remains to show that (5) and (8) are equivalent. Let $E \in G_{k}\left(R^{d}\right)$. There is a neighbourhood $\mathscr{V}(E)$ of $E$ in $G_{k}\left(R^{d}\right)$ and continuous maps $\xi_{1}, \ldots, \xi_{k}$ : $\mathscr{V}(E) \rightarrow R^{d}$ so that for each $V \in \mathscr{V}(E),\left\{\xi_{1}(V), \xi_{2}(V), \ldots, \xi_{k}(V)\right\}$ is a basis for $V$. To do this let $R^{d}=E \oplus E^{\perp}$, and then there is a neighbourhood $\mathscr{V}(E)$ of $E$ such that each $V \in \mathscr{V}(E)$ is the graph of a unique linear map $L_{V}: E \rightarrow E^{\perp}$. Let $\left\{a_{1}, \ldots, a_{k}\right\}$ be a basis for $E$ and then $\left\{\left(a_{1}, L_{V}\left(a_{1}\right)\right),\left(a_{2}, L_{V}\left(a_{2}\right)\right), \ldots,\left(a_{k}, L_{V}\left(a_{k}\right)\right)\right\}$ is a basis for $V$. Since $G_{k}\left(R^{d}\right)$ is compact we can choose a finite collection $\mathscr{V}\left(E_{1}\right), \ldots, \mathscr{V}\left(E_{r}\right)$ of such neighbourhoods that cover $G_{k}\left(R^{d}\right)$, and by disjointifying them we get $v_{1}, \ldots, v_{k}$ : $r^{-1}(k) \rightarrow R^{d}$ defined with the properties in (8). Hence (5) implies (8). If (8) holds then let $x_{0} \in r^{-1}(k)$. If $y \in r^{-1}(k)$, the linear map

$$
L_{y}: \operatorname{span}\left\{u_{1}\left(x_{1}\right), \ldots, u_{k}\left(x_{0}\right)\right\} \rightarrow\left(\operatorname{span}\left\{u_{1}\left(x_{0}\right), \ldots, u_{k}\left(x_{0}\right)\right\}\right)^{\perp}
$$

with graph $\operatorname{span}\left\{u_{1}(y), \ldots, u_{k}(y)\right\}$ depends measurably on $y$, and hence $x \rightarrow$ $V_{x}$ is a measurable map of $r^{-1}(k)$ into $G_{k}\left(R^{d}\right)$.

We say that $\left\{V_{x}\right\}_{x \in X}$ is a measurable subbundle of $X \times R^{d}$ when one, and hence all, of the statements in Theorem 7 hold. Note that if $\left\{U_{x}\right\}_{x \in X},\left\{V_{x}\right\}_{x \in X}$ are measurable subbundles of $X \times R^{d}$ with $U_{x} \subset V_{x}$, and $W_{x}$ is defined by $V_{x}=U_{x} \stackrel{\perp}{\oplus} W_{x}$, using the Euclidean inner-product, then $\left\{W_{x}\right\}_{x \in X}$ is a measurable subbundle.

We can now complete the proof of Lemma 4.

Proof of Lemma 4. We know $\chi: X \times R^{d} \rightarrow R$ is measurable so $\lambda^{(1)}(x)=$ $\sup _{v} \chi(x, v)=\max _{i} \chi\left(x, e_{i}\right)$ is measurable. Consider $\Lambda_{2}=\{(x, v) \in X \times$ $\left.R^{d} \mid \chi(x, v)<\lambda^{(1)}(x)\right\} \in \mathscr{B} \times \mathscr{B}\left(R^{d}\right)$. If $\pi_{1}: X \times R^{d} \rightarrow X$ is the natural projection then by Theorem $6 \pi_{1}\left(\Lambda_{2}\right) \in \mathscr{B}$. Also $\pi_{1}\left(\Lambda_{2}\right)=\{x \mid s(x)>$ 1\}. By Theorem 7 applied to $x \rightarrow \pi_{1}^{-1}(x) \cap \Lambda_{2}$ on $\pi_{1}\left(\Lambda_{2}\right)$ we have measurable maps $r: \pi_{1}\left(\Lambda_{2}\right) \rightarrow \mathbb{N}$ and $u_{1}, \ldots, u_{k}: \pi_{1}\left(\Lambda_{2}\right) \cap r^{-1}(k) \rightarrow R^{d}$ with $u_{1}(x), \ldots, u_{r(x)}(x)$ a basis for $\Lambda_{2} \cap \pi_{1}^{-1}(x)$. Hence $\lambda^{(2)}(x)=\sup \{\chi(x, v) \mid$ $\left.(x, v) \in \Lambda_{2}\right\}=\max _{i} \chi\left(x, u_{i}(x)\right)$ is measurable. Let $\Lambda_{3}=\left\{(x, v) \in X \times R^{d}\right.$ $\left.\chi(x, v)<\lambda^{(2)}(x)\right\}$. As above, we get $\pi_{1}\left(\Lambda_{3}\right) \in \mathscr{B}, \pi_{1}\left(\Lambda_{3}\right)=\{x \mid s(x)>2\}$, and $\lambda^{(3)}$ is measurable. In this way we get that $s: X \rightarrow \mathbb{N}$ is measurable and $\lambda^{(i)}:\{x \mid s(x) \geq i\} \rightarrow R$ is measurable for each $i$. Now

$\left\{(x, v) \mid x \in X, v \in V_{x}^{(j)}\right\}=\left\{(x, v) \in X \times R^{d} \mid \chi(x, v) \leq \lambda^{(j)}(x)\right\} \in \mathscr{B} \times \mathscr{B}\left(R^{d}\right)$

since $\chi$ and $\lambda^{(j)}$ are measurable. By Theorem $7, x \rightarrow V_{x}^{(j)}$ is measurable.

\section{EXISTENCE OF THE LIMIT IN (iv)}

We first note that it suffices to prove part (iv) of Oseledec's theorem when $m$ is an ergodic $T$-invariant measure. To see this suppose (iv) holds whenever $m$ is an ergodic measure and consider

$$
\Delta=\left\{(x, v) \in X \times R^{d} \mid \lim _{n \rightarrow \infty} \frac{1}{n} \log \left\|\left(A^{n}\right)_{x} v\right\| \text { exists }\right\} \in \mathscr{B} \times \mathscr{B}\left(R^{d}\right) .
$$


Let $X^{\prime}=\pi \Delta \backslash \pi\left(\pi^{-1} \pi \Delta \backslash \Delta\right)=\left\{x \in X \mid\{x\} \times R^{d} \subset \Delta\right\}$. Then $X^{\prime} \in \mathscr{B}$ by Theorem 6 and $m\left(X^{\prime}\right)=1$ for every ergodic $T$-invariant measure. Therefore $m\left(X^{\prime}\right)=1$ for every $T$-invariant measure by the ergodic decomposition.

Our first aim is to reduce the problem to a special case by proving Theorem 11. We use the next two simple lemmas to obtain Corollary 10.

Lemma 8. If $T$ is a measure-preserving transformation of a probability space $(X, \mathscr{B}, m)$ and $h: X \rightarrow[0, \infty)$ is measurable then $\liminf _{n \rightarrow \infty} \frac{1}{n} h\left(T^{n} x\right)=0$ a.e.

Proof. Let $A_{k}=\{x \mid h(x) \leq k\}$. THen $\bigcup_{k=1}^{\infty} A_{k}=X$. If $m\left(A_{k}\right)>0$ then, by the recurrence theorem, for a.e. $x \in A_{k}$ there exist $n_{1}(x)<n_{2}(x)<\cdots$ with $T^{n_{i}(x)}(x) \in A_{k} \forall i \geq 1$. Hence $h\left(T^{n_{i}(x)}(x)\right) \leq k$ and so $\liminf _{n \rightarrow \infty} \frac{1}{n} h\left(T^{n} x\right)=$ 0 . This holds for a.e. $x \in \bigcup_{k=1}^{\infty} A_{k}=X$.

For $f: X \rightarrow R, f^{+}: X \rightarrow[0, \infty)$ denotes the positive part of $f$ defined by $f^{+}(x)=\max (0, f(x))$.

Lemma 9. If $T$ is a measure-preserving transformation of a probability space $(X, \mathscr{B}, m)$ and $h: X \rightarrow R$ is measurable and $(h-h \circ T)^{+} \in L^{1}(m)$ then $\frac{1}{n} h\left(T^{n} x\right) \rightarrow 0$ a.e.

Proof. We have $h\left(T^{n} x\right)=h(x)-\sum_{i=0}^{n-1}(h-h \circ T)\left(T^{i} x\right)$. Since $(h-h \circ T)^{+} \epsilon$ $L^{1}(m)$ the ergodic theorem gives $\lim \frac{1}{n} h\left(T^{n} x\right)$ exists a.e. but could take on the value of $\infty$. However the limit is zero by Lemma 8 .

Corollary 10. Suppose $T$ is an ergodic measure-preserving transformation of $(X, \mathscr{B}, m)$ and that $x \rightarrow A_{x}$ is a measurable map of $X$ into $\mathscr{M}(d)$ with $x \rightarrow \log ^{+}\left\|A_{x}\right\|$ integrable. Suppose $\left\{U_{x}\right\}_{x \in X}$ is a measurable subbundle of $X \times R^{d}$ with $A_{x} U_{x} \subset U_{T x}$. By the subadditive ergodic theorem we have

$$
\lim _{n \rightarrow \infty} \frac{1}{n} \log \left\|\left.A^{n}\right|_{U_{x}}\right\|
$$

exists and is constant a.e., but could be $-\infty$, and suppose the value of this limit is less than or equal to $\rho \in R$. For $\varepsilon>0$ define

$$
a_{\varepsilon}(x)=\sup _{n \geq 0}\left(\left\|\left.A^{n}\right|_{U_{x}}\right\| e^{-n(\rho+\varepsilon)}\right) .
$$

Then $\frac{1}{n} \log a_{\varepsilon}\left(T^{n} x\right) \rightarrow 0$ a.e.

Proof. By the choice of $\rho$ we have $1 \leq a_{\varepsilon}(x)<\infty$. Also

$$
\frac{a_{\varepsilon}(x)}{a_{\varepsilon}(T x)} \leq \max \left(\left\|\left.A\right|_{U_{x}}\right\| e^{-(\rho+\varepsilon)}, 1\right)
$$

so that

$$
\log a_{\varepsilon}(x)-\log a_{\varepsilon}(T x) \leq \max \left(\log ^{+}\left\|A_{x}\right\|-(\rho+\varepsilon), 0\right) .
$$

Hence $x \rightarrow\left(\log a_{\varepsilon}(x)-\log a_{\varepsilon}(T x)\right)^{+}$is integrable and we can apply Lemma 9.

We shall mostly use the next result with $U_{x}=V_{x}^{(i+1)}, V_{x}=V_{x}^{(i)}$ for some $i$, but we consider a more general situation in order to prove Corollary 12 . 
Theorem 11. Let $T$ be an ergodic measure-preserving transformation of the probability space $(X, \mathscr{B}, m)$ and let $A: X \rightarrow \mathscr{M}(d)$ be such that $x \rightarrow \log ^{+}\left\|A_{x}\right\|$ is integrable. Assume $\left\{U_{x}\right\}_{x \in X},\left\{V_{x}\right\}_{x \in X}$ are measurable subbundles of $X \times R^{d}$ with $U_{x} \subset V_{x}, A_{x} U_{x} \subset U_{T x}$, and $A_{x} V_{x} \subset V_{T x} \forall x \in X$. Let $Y \in \mathscr{B}$ be a set of full measure with $T Y \subset Y$ and let $\rho, \lambda$ be real numbers where

$$
\limsup _{n \rightarrow \infty} \frac{1}{n} \log \left\|\left(A^{n}\right)_{x} u\right\| \leq \rho \quad \forall u \in U_{x} \backslash\{0\} \quad \forall x \in Y
$$

and

$$
\limsup _{n \rightarrow \infty} \frac{1}{n} \log \left\|\left(A^{n}\right)_{x} v\right\|=\lambda \quad \forall v \in V_{x} \backslash U_{x} \quad \forall x \in Y
$$

Assume $\rho<\lambda$. Define the measurable subbundle $\left\{W_{x}\right\}_{x \in X}$ by $V_{x}=U_{x} \stackrel{\perp}{\oplus} W_{x}$ using the Euclidean inner-product on $R^{d}$. Let $\left.A_{x}\right|_{V_{x}}: V_{x} \rightarrow V_{T x}$ induce the linear maps $C_{x}: W_{x} \rightarrow W_{T x}, B_{x}: W_{x} \rightarrow U_{T x}$ by $A_{x}(w)=B_{x}(w) \stackrel{\perp}{\oplus} C_{x}(w)$. Then there exists $Y^{\prime} \in \mathscr{B}$ with $m\left(Y^{\prime}\right)=1$ and $T Y^{\prime} \subset Y^{\prime}$ with the following properties: We have

$$
\begin{aligned}
& \limsup _{n \rightarrow \infty} \frac{1}{n} \log \left\|\left(C^{n}\right)_{x} w\right\|=\limsup _{n \rightarrow \infty} \frac{1}{n} \log \left\|\left(A^{n}\right)_{x}(u \oplus w)\right\| \\
& \forall w \in W_{x} \backslash\{0\}, \forall u \in U_{x}, \quad \forall x \in Y^{\prime} .
\end{aligned}
$$

Moreover, if $\lim _{n \rightarrow \infty} \frac{1}{n} \log \left\|\left(C^{n}\right)_{x} w\right\|$ exists for some $w \in W_{x} \backslash\{0\}$ and some $x \in Y^{\prime}$ then $\lim _{n \rightarrow \infty} \frac{1}{n} \log \left\|\left(A^{n}\right)_{x}(u \oplus w)\right\|$ exists $\forall u \in U_{x}$ and equals $\lim _{n \rightarrow \infty} \frac{1}{n} \log \left\|\left(C^{n}\right)_{x} w\right\|$.

Proof. We use $u$ to denote a general element of some $U_{x}$, and $w$ to denote a general element of some $W_{x}$. We have $\left(A^{n}\right)_{x}(u \oplus w)=\left[\left(A^{n}\right)_{x} u+D_{x}^{(n)} w\right] \oplus$ $\left(C^{n}\right)_{x} w$, where $D_{x}^{(n)}: W_{x} \rightarrow U_{T^{n} x}$ is $\sum_{i=0}^{n-1}\left(A^{n-i-1}\right)_{T^{i+1} x} B_{T^{i} x}\left(C^{i}\right)_{x}$. Taking the square of the Euclidean norm of each side and using Lemma 1 we get

$$
\begin{aligned}
& \underset{n \rightarrow \infty}{\limsup } \frac{1}{n} \log \left\|\left(A^{n}\right)_{x}(u \oplus w)\right\| \\
& \quad=\max \left(\limsup _{n \rightarrow \infty} \frac{1}{n} \log \left\|\left(A^{n}\right)_{x} u+D_{x}^{(n)} w\right\|, \limsup _{n \rightarrow \infty} \frac{1}{n} \log \left\|\left(C^{n}\right)_{x} w\right\|\right) .
\end{aligned}
$$

Putting $u=0$ and $w \neq 0$ gives

$$
\begin{aligned}
& \limsup _{n \rightarrow \infty} \frac{1}{n} \log \left\|\left(A^{n}\right)_{x} w\right\| \\
& \quad=\max \left(\limsup \frac{1}{n} \log \left\|D_{x}^{(n)} w\right\|, \lim \sup \frac{1}{n} \log \left\|\left(C^{n}\right)_{x} w\right\|\right) .
\end{aligned}
$$

For $\varepsilon>0$ let $a_{\varepsilon}(x)=\sup _{n \geq 0}\left(\left\|\left.A^{n}\right|_{U_{x}}\right\| e^{-n(\rho+\varepsilon)}\right)$. Let $\left\{\varepsilon_{p}\right\}_{p=1}^{\infty}$ be a sequence with $\varepsilon_{p} \searrow 0$ and, using Corollaries 9 and 10, let $Y_{p}$ be the subset of $Y$ of full measure on which $\frac{1}{n} \log a_{\varepsilon_{p}}\left(T^{n} x\right) \rightarrow 0$ and $\frac{1}{n} \log ^{+}\left\|A_{T^{n} x}\right\| \rightarrow 0 \forall x \in Y_{p}$. We shall show $\forall x \in \bigcap_{p=1}^{\infty} Y_{p}$,

$$
\limsup _{n \rightarrow \infty} \frac{1}{n} \log \left\|\left(C^{n}\right)_{x} w\right\|=\limsup _{n \rightarrow \infty} \frac{1}{n} \log \left\|\left(A^{n}\right)_{x} w\right\|, \quad \forall w \neq 0 .
$$


If not, choose $x \in \bigcap_{p=1}^{\infty} Y_{p}$ and $w \neq 0$ with

$$
\limsup _{n \rightarrow \infty} \frac{1}{n} \log \left\|\left(C^{n}\right)_{x} w\right\|=\tau<\lambda^{\prime}=\limsup _{n \rightarrow \infty} \frac{1}{n} \log \left\|\left(A^{n}\right)_{x} w\right\| .
$$

Choose $p$ so that $\max (\tau, \rho)+\varepsilon_{p}<\lambda^{\prime}$. There exists $N$, depending on $x, w$, and $p$, such that $n \geq N$ implies $\left\|\left(C^{n}\right)_{x} w\right\|<e^{n\left(\tau+\varepsilon_{p}\right)}$. If we write $L_{x}: U_{x} \rightarrow$ $U_{T x}$ instead of $\left.A_{x}\right|_{U_{x}}$ then

$$
\begin{aligned}
\left\|D_{x}^{(n)} w\right\| & \leq \sum_{i=0}^{n-1}\left\|\left(L^{n-i-1}\right)_{T^{i+1} x}\right\| \cdot\left\|B_{T^{i x}}\right\| \cdot\left\|\left(C^{i}\right)_{x} w\right\| \\
& \leq n \max _{0 \leq i \leq n-1}\left\|\left(L^{n-i-1}\right)_{T^{i+1} x}\right\| \cdot\left\|B_{T^{i} x}\right\| \cdot\left\|\left(C^{i}\right)_{x} w\right\| \\
& =n\left\|\left(L^{n-i_{n}-1}\right)_{T^{i_{n}+1} x}\right\| \cdot\left\|B_{T^{i_{n}} x}\right\| \cdot\left\|\left(C^{i_{n}}\right)_{x} w\right\|
\end{aligned}
$$

for some $0 \leq i_{n} \leq n-1$, which depends on $x$ and $w$. Note that $\left\{i_{n}\right\}$ is an increasing sequence. If $\left\{i_{n}\right\}$ is unbounded then $i_{n} \geq N$ eventually and

$$
\begin{aligned}
\frac{1}{n} \log \left\|D_{x}^{(n)} w\right\| \leq & \frac{1}{n} \log n+\frac{1}{n} \log a_{\varepsilon_{p}}\left(T^{1+i_{n}} x\right)+\frac{\left(n-1-i_{n}\right)}{n}\left(\rho+\varepsilon_{p}\right) \\
& +\frac{1}{n} \log ^{+}\left\|B_{T^{i_{x}}}\right\|+\frac{i_{n}}{n}\left(\tau+\varepsilon_{p}\right)
\end{aligned}
$$

so $\lim \sup _{n \rightarrow \infty} \frac{1}{n} \log \left\|D_{x}^{(n)} w\right\| \leq \max (\tau, \rho)+\varepsilon_{p}<\lambda^{\prime}$, which contradicts (**). Here we used

$$
\frac{1}{n} \log ^{+}\left\|B_{T^{i_{n}}}\right\| \leq \frac{i_{n}}{n} \frac{1}{i_{n}} \log ^{+}\left\|A_{T^{i_{n}} x}\right\| \rightarrow 0 .
$$

If $\left\{i_{n}\right\}$ is bounded, say $i_{n} \leq M$ for all $n$, then

$$
\begin{aligned}
\frac{1}{n} \log \left\|D_{x}^{(n)} w\right\| \leq & \frac{1}{n} \log n+\max _{0 \leq i \leq M} \frac{1}{n} \log \left\|\left(L^{n-i-1}\right)_{T^{i+1} x}\right\| \\
& +\max _{0 \leq i \leq M} \frac{1}{n} \log \left\|B_{T^{i} x}\right\|+\max _{0 \leq i \leq M} \frac{1}{n} \log \left\|\left(C^{i}\right)_{x} w\right\|,
\end{aligned}
$$

so that $\lim \sup _{n \rightarrow \infty} \frac{1}{n} \log \left\|D_{x}^{(n)} w\right\| \leq \rho$, which contradicts $(* *)$. Hence

$$
\begin{aligned}
& \limsup _{n \rightarrow \infty} \frac{1}{n} \log \left\|\left(C^{n}\right)_{x} w\right\|=\limsup _{n \rightarrow \infty} \frac{1}{n} \log \left\|\left(A^{n}\right)_{x} w\right\| \\
& \qquad \forall x \in \bigcap_{p=1}^{\infty} Y_{p} \equiv Y^{\prime} \forall w \in W_{x} \backslash\{0\} .
\end{aligned}
$$

By $(*)$ and the above reasoning this also equals

$$
\limsup _{n \rightarrow \infty} \frac{1}{n} \log \left\|\left(A^{n}\right)_{x}(u \oplus w)\right\| \quad \forall u \in U_{x} .
$$

Now suppose $\lim _{n \rightarrow \infty} \frac{1}{n} \log \left\|\left(C^{n}\right)_{x} w\right\|$ exists for some $w \in W_{x} \backslash\{0\}$ and some $x \in Y^{\prime}$. By Lemma 1 we have, using the Euclidean norm,

$$
\begin{aligned}
\liminf _{n \rightarrow \infty} & \frac{1}{n} \log \left\|\left(A^{n}\right)_{x}(u \oplus w)\right\| \\
\geq & \max \left(\liminf _{n \rightarrow \infty} \frac{1}{n} \log \left\|\left(A^{n}\right)_{x} u+D_{x}^{(n)} w\right\|, \liminf _{n \rightarrow \infty} \frac{1}{n} \log \left\|\left(C^{n}\right)_{x} w\right\|\right) \\
\geq & \lim _{n \rightarrow \infty} \frac{1}{n} \log \left\|\left(C^{n}\right)_{x} w\right\| .
\end{aligned}
$$


With the first part of the proof this gives that $\lim _{n \rightarrow \infty} \frac{1}{n} \log \left\|\left(A^{n}\right)_{x}(u \oplus w)\right\|$ exists $\forall u \in U_{x}$ and equals $\lim _{n \rightarrow \infty} \frac{1}{n} \log \left\|\left(C^{n}\right)_{x} w\right\|$.

If we consider Theorem 11 with $U_{x}=V_{x}^{(i+1)}, V_{x}=V_{x}^{(i)}$ for some $i$ then we can consider the induced map $C_{x}: W_{x} \rightarrow W_{T_{x}}$ where $V_{x}^{(i)}=V_{x}^{(i+1)} \stackrel{\frac{1}{\oplus}}{\oplus} W_{x}$ and we have $\lim \sup _{n \rightarrow \infty} \frac{1}{n} \log \left\|\left(C^{n}\right)_{x} w\right\|=\lambda^{(i)} \forall w \in W_{x} \backslash\{0\}$. Therefore under $\left\{C_{x}\right\}$ every nonzero vector gives the same value $\lambda^{(i)}$ for the limit supremum. If we could replace "lim sup" by "lim" for $\left\{C_{x}\right\}$ then, by the last part of Theorem 11 , we could conclude $\lim _{n \rightarrow \infty} \frac{1}{n} \log \left\|\left(A^{n}\right)_{x} v\right\|$ exists $\forall v \in V_{x}^{(i)} \backslash V_{x}^{(i+1)}$. So we can reduce our problem to considering a family $\left\{C_{x}\right\}$ for which the lim sup takes the same value for all nonzero vectors. We shall deal with this case by considering the map induced on $X \times P\left(R^{d}\right)$ and using some "relative" ergodic theory. However we consider the ergodic theory of the map induced on $X \times$ $P\left(R^{d}\right)$ in the general case $\left\{A_{x}\right\}$ as this throws light on this situation. To define the map on $X \times P\left(R^{d}\right)$ we need each $A_{x}$ to be invertible:

Corollary 12. Let $T$ be ergodic. To prove Oseledec's theorem for the case when each $A_{x} \in \mathscr{M}(d)$ and $x \rightarrow \log ^{+}\left\|A_{x}\right\|$ is integrable it suffices to prove it for the case when $A_{x} \in G L(d)$ a.e. and $x \rightarrow \log ^{+}\left\|A_{x}\right\|$ is integrable.

Proof. If $A_{x} \notin G L(d)$ then $A_{x} v=0$ for some $v \neq 0$ so $\lambda^{(s(x))}(x)=-\infty$. If this occurs on a set of positive measure then $\lambda^{(s(x))}(x)=-\infty$ a.e. Define the measurable subbundle $\left\{W_{x}\right\}_{x \in X}$ by $W_{x}=\left(V_{x}^{(s(x))}\right)^{\perp}$ and consider the linear map $C_{x}: W_{x} \rightarrow W_{T x}$ defined by letting $C_{x} w$ be the orthogonal projection of $A_{x} w$ onto $W_{T x}$. Then $x \rightarrow \log ^{+}\left\|C_{x}\right\|$ is integrable and each $C_{x}$ is invertible since

$$
\limsup _{n \rightarrow \infty} \frac{1}{n} \log \left\|\left(C^{n} x\right) w\right\| \neq-\infty \quad \text { a.e. }
$$

by Theorem 11. The Lyapunov exponents for $x \rightarrow C_{x}$ are $\lambda^{(s(x)-1)}(x), \ldots$, $\lambda^{(1)}(x)$ and the corresponding filtration is $V_{x}^{(s(x)-1)} \cap W_{x}, \ldots, V_{x}^{(1)} \cap W_{x}=W_{x}$, by Theorem 11. Suppose we have proved Oseledec's theorem for $x \rightarrow C_{x}$, so that $\lim _{n \rightarrow \infty} \frac{1}{n} \log \left\|\left(C^{n}\right)_{w} w\right\|=\lambda^{(j)}(x)$ for a.e. $x$ and all

$$
w \in\left(V_{x}^{(j)} \cap W_{x}\right) \backslash\left(V_{x}^{(j+1)} \cap W_{x}\right)=\left(V_{x}^{(j)} \backslash V_{x}^{(j+1)}\right) \cap W_{x} .
$$

Then by the second part of Theorem $11 \lim _{n \rightarrow \infty} \frac{1}{n} \log \left\|\left(A^{n}\right)_{x} v\right\|=\lambda^{(j)}(x)$ for a.e. $x$ and all $v \in V_{x}^{(j)} \backslash V_{x}^{(j+1)}$ and Oseledec's theorem holds for $x \rightarrow A_{x}$.

From now on we assume $A_{x} \in G L(d) \forall x \in X$. We can then define $\varphi: X \times$ $P\left(R^{d}\right) \rightarrow R$ by $\varphi(x, u)=\log \left(\left\|A_{x} \tilde{u}\right\| /\|\tilde{u}\|\right)$ where $\tilde{u}$ is any nonzero element of $R^{d}$ on the line in $R^{d}$ representing $u \in P\left(R^{d}\right)$. The function $\varphi$ is measurable and for each $x \in X$ the map $u \rightarrow \varphi(x, u)$ is a real-valued continuous map of $P\left(R^{d}\right)$. If we define $\Phi: X \rightarrow R$ by $\Phi(x)=\sup _{u \in P\left(R^{d}\right)} \varphi(x, u)$ then $\Phi(x)=$ $\log \left\|A_{x}\right\|$ so the condition that $x \rightarrow \log ^{+}\left\|A_{x}\right\|$ be integrable is equivalent to $x \rightarrow$ $\Phi^{+}(x) \equiv \max (0, \Phi(x))$ being integrable. Define $S: X \times P\left(R^{d}\right) \rightarrow X \times P\left(R^{d}\right)$ by $S(x, u)=\left(T x, A_{x} u\right)$ where $A_{x}: P\left(R^{d}\right) \rightarrow P\left(R^{d}\right)$ is the homeomorphism induced by $A_{x}: R^{d} \rightarrow R^{d}$. If $\tilde{u}$ is any nonzero element of $R^{d}$ on the line 
given by $u \in P\left(R^{d}\right)$ we have

$$
\sum_{i=0}^{n-1} \varphi\left(S^{i}(x, u)\right)=\log \left\|\left(A^{n}\right)_{x} \tilde{u}\right\|-\log \|\tilde{u}\|
$$

so

$$
\limsup _{n \rightarrow \infty} \frac{1}{n} \sum_{i=0}^{n-1} \varphi\left(S^{i}(x, u)\right)=\limsup _{n \rightarrow \infty} \frac{1}{n} \log \left\|\left(A_{x}^{n}\right) \tilde{u}\right\|
$$

and if one side has a limit so does the other.

In the proof of the next result we shall use the following fact about dual spaces. Let $(X, \mathscr{B}, m)$ be a probability space and let $E$ be a separable Banach space with dual space $E^{*}$. Let $L_{m}^{1}(E)$ be the space of all measurable functions $f: X \rightarrow E$ with $x \rightarrow\|f(x)\|$ integrable where two such functions are identified if they are equal a.e. This is a Banach space with norm $\|f\|=\int\|f(x)\| d m(x)$. Let $L_{m}^{\infty}\left(E^{*}, E\right)$ be the space of all maps $\gamma: X \rightarrow E^{*}$ for which $x \rightarrow \gamma_{x}(v)$ is bounded and measurable for each $v \in E$, where two such functions $\gamma^{(1)}, \gamma^{(2)}$ are identified if $x \rightarrow \gamma_{x}^{(1)}(v)$ is equal a.e. to $x \rightarrow \gamma_{x}^{(2)}(v)$ for every $v \in E$. This is a Banach space with norm $\|\gamma\|_{\infty}=$ ess.sup. $\|\gamma(x)\|$, which is finite by the principle of uniform boundedness. Then the map $\Psi: L_{m}^{\infty}\left(E^{*}, E\right) \rightarrow\left(L_{m}^{1}(E)\right)^{*}$, given by $(\Psi \gamma)(f)=\int \gamma_{x}\left(f_{x}\right) d m(x)$ where $\gamma: X \rightarrow E^{*}\left(x \rightarrow \gamma_{x}\right)$ is in $L_{m}^{\infty}\left(E^{*}, E\right)$ and $f: X \rightarrow E\left(x \rightarrow f_{x}\right)$ is in $L_{m}^{1}(E)$, is an isometric isomorphism of Banach spaces [1, p. 47; 11, p. 95]. We shall be interested in the case when $E=C(P, R)$ for a compact metric space $P$. The set $L_{m}(M(P))$ of all measurable maps from $X$ to $M(P)$ is a subset of the unit ball in $L_{m}^{\infty}\left(E^{*}, E\right)$, with $E=C(P, R)$, and is closed with respect to the weak*-topology on $L_{m}^{\infty}\left(E^{*}, E\right)$. Hence $L_{m}(M(P))$ is compact with respect to this topology. The set $L_{m}(M(P))$ can be identified with $M_{m}(X \times P)=\{\nu \in M(X \times P) \mid \nu$ projects to $m$ on $X\}$ via the map $\alpha \rightarrow \bar{\alpha} \in M_{m}(X \times P)$ where for $x \rightarrow f_{x}$ in $L_{m}^{1}(C(P, R))$ we have

$$
\int_{X \times P} f_{x}(y) d \bar{\alpha}(x, y)=\int_{X}\left(\int_{P} f_{x}(y) d \alpha_{x}(y)\right) d m(x) .
$$

The map $\varphi: X \times P\left(R^{d}\right) \rightarrow R$, defined above, gives a map $x \rightarrow \varphi(x,$.$) of X$ into $C\left(P\left(R^{d}\right), R\right)$. We noted above that $\sup \left\{\varphi(x, u) \mid u \in P\left(R^{d}\right)\right\}=\log \left\|A_{x}\right\|$ and an easy calculation gives $\inf \left\{\varphi(x, u) \mid u \in P\left(R^{d}\right)\right\}=-\log \left\|\left(A_{x}\right)^{-1}\right\|$. Hence $\log ^{+}\left\|\left(A_{x}\right)^{-1}\right\|=-\min \left(0, \inf \left\{\varphi(x, u) \mid u \in P\left(R^{d}\right)\right\}\right.$. Therefore $x \rightarrow \varphi(x,)$. is in $L_{m}^{1}\left(C\left(P\left(R^{d}\right), R\right)\right)$ iff both $x \rightarrow \log ^{+}\left\|A_{x}\right\|$ and $x \rightarrow \log ^{+}\left\|\left(A_{x}\right)^{-1}\right\|$ are integrable iff both $x \rightarrow \log \left\|A_{x}\right\|$ and $x \rightarrow \log \left\|\left(A_{x}\right)^{-1}\right\|$ are integrable. It is for this reason we have to assume both of these integrability conditions in the last stage of the proof.

A version of the following result appears in [4].

Theorem 13. Let $T$ be an ergodic measure-preserving transformation of a probability space $(X, \mathscr{B}, m)$. Let $P$ be a compact metric space and let $S: X \times P \rightarrow$ $X \times P$ be a measurable map of the form $S(x, u)=\left(T x, S_{x} u\right)$ where, for each $x \in X$, the map $S_{x}: P \rightarrow P$ is continuous. Let $\varphi: X \times P \rightarrow R$ be measurable and for every $x \in X$ let $\varphi(x,):. P \rightarrow R$ be continuous. If $\Phi: X \rightarrow R$ is defined 
by $\Phi(x)=\sup _{u \in P} \varphi(x, u)$ then suppose $\Phi^{+} \in L_{m}^{1}$. Then for $m$ a.e. $x \in X$,

$$
\lim _{n \rightarrow \infty} \sup _{u \in P} \frac{1}{n} \sum_{i=0}^{n-1} \varphi\left(S^{i}(x, u)\right)=\sup \left\{\int \varphi d \nu \mid \nu \in M_{m}(X \times P, S)\right\} .
$$

Proof. Note that the extreme points of the convex set $M_{m}(X \times P, S)$, of all $S$-invariant members of $M_{m}(X \times P)$, are exactly the ergodic members of $M_{m}(X \times P, S)$.

Note that if $\psi: X \times P \rightarrow R$ is measurable and $\psi(x,):. P \rightarrow R$ is continuous for each $x \in X$ then $x \rightarrow \sup _{u \in P} \psi(x, u)$ is measurable since if $\left\{u_{n}\right\}_{n=1}^{\infty}$ is a dense subset of $P$ then $\sup _{u \in P} \psi(x, u)=\sup _{n \geq 1} \psi\left(x, u_{n}\right)$. Let

$$
M_{n}(x)=\sup _{u \in P} \sum_{i=0}^{n-1} \varphi\left(S^{i}(x, u)\right) .
$$

This is measurable and $M_{1}^{+} \in L_{m}^{1}$ so the subadditive ergodic theorem gives that $\lim _{n \rightarrow \infty} \frac{1}{n} M_{n}(x)$ exists a.e. and is equal to a constant $c$ a.e. If we put $b=\sup \left\{\int \varphi d \nu \mid \nu \in M_{m}(X \times P, S)\right\}$ then we have to show $c=b$. Note that $c, b$ could equal $-\infty$.

To show $c \geq b$ is easy. Suppose $b \neq-\infty$, or there is nothing to prove. For each $\varepsilon>0$ we can choose an ergodic $\nu_{\varepsilon} \in M_{m}(X \times P, S)$ with $\int \varphi d \nu_{\varepsilon}>b-\varepsilon$. By Birkhoff's ergodic theorem

$$
\frac{1}{n} \sum_{i=0}^{n-1} \varphi\left(S^{i}(x, u)\right) \rightarrow \int \varphi d \nu_{\varepsilon}
$$

for $\nu_{\varepsilon}$ a.e. $(x, u) \in X \times P$. But $M_{n}(x) \geq \sum_{i=0}^{n-1} \varphi\left(S^{i}(x, u)\right)$ so $c \geq b-\varepsilon$.

In order to show $c \leq b$ consider, for each $n \geq 1$, the set

$$
\Delta_{n}=\left\{(x, u) \in X \times P \mid \sum_{i=0}^{n-1} \varphi\left(S^{i}(x, u)\right)=M_{n}(x)\right\} \in \mathscr{B} \times \mathscr{B}(P) .
$$

We have $\pi \Delta_{n}=X$, where $\pi: X \times P \rightarrow X$ is the natural projection. Since for each $x,\left\{u \in P \mid(x, u) \in \Delta_{n}\right\}$ is closed we can choose a measurable map $w_{n}: X \rightarrow P$ with $\left(x, w_{n}(x)\right) \in \Delta_{n} \forall x \in X$ (Theorem 5). Hence $M_{n}(x)=$ $\sum_{i=0}^{n-1} \varphi\left(S^{i}\left(x, w_{n}(x)\right)\right.$ and $x \rightarrow \delta_{w_{n}(x)}$ is in $L_{m}(M(P))$. For each $n \geq 1$ the linear functional on $L_{m}^{1}(C(P, R))$ given by

$$
\psi \rightarrow \frac{1}{n} \sum_{i=0}^{n-1} \int \psi S^{i}\left(x, w_{n}(x)\right) d m(x)
$$

gives an element $\alpha^{(n)}$ of $L_{m}(M(P))$. The sequence $\left\{\alpha^{(n)}\right\}$ has a convergent subsequence in the weak*-topology. Therefore there is $n_{j} \nearrow \infty$ and $\alpha \in L_{m}(M(P))$, which corresponds to some $\bar{\alpha} \in M_{m}(X \times P)$, with

$$
\int \frac{1}{n_{j}} \sum_{i=0}^{n_{j}-1} \psi S^{i}\left(x, w_{n_{j}}(x)\right) d m(x) \rightarrow \int \psi d \bar{\alpha} \quad \forall \psi \in L_{m}^{1}(C(P, R)) .
$$

If, for each $N \geq 1$, we let $\varphi_{N}=\max (\varphi,-N)$ then $\varphi_{N} \in L_{m}^{1}(C(P, R))$ and $M_{n_{j}}(x) \leq \sum_{i=0}^{n_{j}-1} \varphi_{N} S^{i}\left(x, w_{n_{j}}(x)\right)$. Hence $c \leq \int \varphi_{N} d \bar{\alpha}$ for each $N$ and hence 
$c \leq \int \varphi d \bar{\alpha}$. We now show $\bar{\alpha} \in M_{m}(X \times P, S)$ and this gives $c \leq b$. If $\psi \in L_{m}^{1}(C(P, R))$ then

$$
\begin{aligned}
& \left|\int \psi \circ S d \bar{\alpha}-\int \psi d \bar{\alpha}\right| \\
& \quad=\lim _{j \rightarrow \infty} \frac{1}{n_{j}}\left|\int\left[\psi\left(S^{n_{j}}\left(x, w_{n_{j}}(x)\right)\right)-\psi\left(x, w_{n_{j}}(x)\right)\right] d m(x)\right| \\
& \quad \leq \lim _{j \rightarrow \infty} \frac{1}{n_{j}} 2 \int\|\psi(x, .)\| d m(x)=0 .
\end{aligned}
$$

Hence $\bar{\alpha}$ is $S$-invariant.

Corollary 14. Suppose in addition to the assumptions of Theorem 13 that $\int \varphi d \nu$ takes the same value $b$ for all $\nu \in M_{m}(X \times P, S)$ and that $x \rightarrow \inf _{u \in P} \varphi(x, u)$ is also in $L_{m}^{1}$. Then for $m$ a.e. $x \in X, \frac{1}{n} \sum_{i=0}^{n-1} \varphi\left(S^{i}(x, u)\right) \rightarrow b$ uniformly in $u \in P$.

Proof. By Theorem 13 we have

$$
\lim _{n \rightarrow \infty} \sup _{u \in P} \frac{1}{n} \sum_{i=0}^{n-1} \varphi\left(S^{i}(x, u)\right) \rightarrow b \quad \forall x \in X^{\prime}
$$

with $m\left(X^{\prime}\right)=1$. By applying Theorem 13 to $-\varphi$ instead of $\varphi$ we get

$$
\lim _{n \rightarrow \infty} \inf _{u \in P} \frac{1}{n} \sum_{i=0}^{n-1} \varphi\left(S^{i}(x, u)\right) \rightarrow b \quad \forall x \in X^{\prime \prime}
$$

with $m\left(X^{\prime \prime}\right)=1$. Hence for $x \in X^{\prime} \cap X^{\prime \prime}, \frac{1}{n} \sum_{i=0}^{n-1} \varphi\left(S^{i}(x, u)\right) \rightarrow b$ uniformly in $u \in P$.

We now complete the proof of Osedelec's theorem. Because

$$
x \rightarrow \log ^{+}\left\|\left(A_{x}\right)^{-1}\right\|
$$

is integrable we have $\lambda^{(s(x))}(x) \neq-\infty$ a.e. By Lemma 4 and the ergodicity of $T$ there is a set $X_{3} \in \mathscr{B}$ with $m\left(X_{3}\right)=1, T X_{3} \subset X_{3}$ on which $x \rightarrow s(x)$ is constant, each $x \rightarrow \lambda^{(i)}(x)$ is constant and each $x \rightarrow \operatorname{dim}\left(V_{x}^{(i)}\right)$ is constant. By Lemma 4(iii) and Theorem 7 there is a bimeasurable bijection between $\left\{V_{x}^{(s)}\right\}_{x \in X_{3}}$ and $X_{3} \times R^{r_{s}}$ covering the identity and which is linear on fibres; where $r_{s}=\operatorname{dim}\left(V_{x}^{(s)}\right)$. Using this bijection we can define $\varphi: X_{3} \times R^{r_{s}} \rightarrow R$ by $\varphi(x, u)=\log \left(\left\|A_{x} \tilde{u}\right\| /\|\tilde{u}\|\right.$ where $\tilde{u}$ is any nonzero element of $R^{r_{s}}$ on the line given by $u$. We have $\lim \sup _{n \rightarrow \infty} \frac{1}{n} \log \left\|\left(A^{n}\right)_{x} v\right\|=\lambda^{(s)} \forall v \in V_{x}^{(s)} \backslash\{0\}$ so we have

$$
\limsup _{n \rightarrow \infty} \frac{1}{n} \sum_{i=0}^{n-1} \varphi\left(S^{i}(x, u)\right)=\lambda^{(s)} \quad \forall u \in P\left(R^{r_{s}}\right) .
$$

By Birkhoff's ergodic theorem we have $\int \varphi d \nu=\lambda^{(s)} \forall \nu \in M_{m}\left(X_{3} \times P\left(R^{r_{s}}\right), S\right)$. Because we are assuming $x \rightarrow \log ^{+}\left\|\left(A_{x}\right)^{-1}\right\|$ is integrable, the conditions of Corollary 14 hold and we get for all $x \in X_{4}$, where $m\left(X_{4}\right)=1$ and $T X_{4} \subset X_{4}$, $\frac{1}{n} \sum_{i=0}^{n-1} \varphi\left(S^{i}(x, u)\right)=\lambda^{(s)}$ uniformly in $u \in P\left(R^{r_{s}}\right)$. This says $\frac{1}{n} \log \left\|\left(A^{n}\right)_{x} v\right\| \rightarrow$ $\lambda^{(s)} \forall v \in V_{x}^{(s)} \backslash\{0\}$ (and uniformly over $\left\{v \in V_{x}^{s} \mid\|v\|=1\right\}$ ). 
Now consider $\left\{V_{x}^{(s-1)}\right\}_{x \in X_{4}}$. Write $V_{x}^{(s-1)}=V_{x}^{s} \stackrel{\perp}{\oplus} W_{x}$. By Theorem 11 we have for a.e. $x$,

$$
\limsup _{n \rightarrow \infty} \frac{1}{n} \log \left\|\left(C_{x}^{n}\right) w\right\|=\lambda^{(s-1)} \quad \forall w \in W_{x} \backslash\{0\},
$$

where $C_{x}: W_{x} \rightarrow W_{T x}$ is induced from $A_{x}$. Since $\left\{W_{x}\right\}$ is a measurable subbundle and $\left\|C_{x}\right\| \leq\left\|A_{x}\right\|,\left\|\left(C_{x}\right)^{-1}\right\| \leq\left\|\left(A_{x}\right)^{-1}\right\|$ we can apply the above reasoning to $x \rightarrow C_{x}$ on $\left\{W_{x}\right\}$. This gives for a.e. $x$ that $\frac{1}{n} \log \left\|\left(C^{n}\right)_{x} w\right\| \rightarrow$ $\lambda^{(s-1)} \forall w \in W_{x} \backslash\{0\}$. Then by the second part of Theorem 11 we get for a.e. $x$,

$$
\frac{1}{n} \log \left\|\left(A^{n}\right)_{x} v\right\| \rightarrow \lambda^{(s-1)} \quad \forall v \in V_{x}^{(s-1)} \backslash V_{x}^{s} .
$$

The proof follows by repeating the above reasoning.

Note that for $\varphi: X \rightarrow R^{d} \rightarrow R$ defined by $\varphi(x, u)=\log \left(\left\|A_{x} \tilde{u}\right\| /\|\tilde{u}\|\right)$ we have for almost every $x \in X$,

$$
\frac{1}{n} \sum_{i=0}^{n-1} \varphi\left(S^{i}(x, u)\right) \rightarrow \lambda^{(i)} \quad \forall u \in P\left(V_{x}^{(i)}\right) \backslash P\left(V_{x}^{(i+1)}\right) .
$$

For each ergodic $\nu \in M_{m}(X \times P, S)$ there is a largest $i$ with $\nu\left(\bigcup_{x} P\left(V_{x}^{(i)}\right)\right)=1$, and for this $i, \int \varphi d \nu=\lambda^{(i)}$. Moreover, for each $i$ there exists an ergodic measure corresponding to $i$ in the above way. Hence the exponents are the values of $\int \varphi d \nu$ as $\nu$ runs over ergodic measures in $M_{m}(X \times P, S)$. This was first pointed out by Ledrappier [6].

\section{REFERENCES}

1. N. Bourbaki, Intégration, Vol. VI, Chapitre 6, Hermann, Paris, 1959.

2. C. Castaing and M. Valadier, Convex analysis and measurable multifunctions, Lecture Notes in Math., Vol. 580, Springer-Verlag, Berlin and New York, 1975.

3. J. E. Cohen, H. Kesten, and C. M. Newman (eds.), Random matrices and their applications, Comtemp. Math., Vol. 50, Amer. Math. Soc., Providence, R. I., 1984.

4. H. Crauel, Lyapunov exponents and invariant measures of stochastic systems on manifolds, Lecture Notes in Math., Vol. 1186, Springer-Verlag, Berlin and New York, 1985, pp. 271291.

5. M. W. Hirsch, Differential topology, Graduate Texts in Math., Vol. 33, Springer-Verlag, Berlin and New York, 1976.

6. F. Ledrappier, Quelques proprietes des exposants caracteristiques, Lecture Notes in Math., Vol. 1098, Springer-Verlag, Berlin and New York, 1984, pp. 306-396.

7. V. I. Oseledec, A multiplicative ergodic theorem and Lyapunov characteristic numbers for dynamical systems, Trans. Moscow Math. Soc. 19 (1968), 197-231.

8. Ya. B. Pesin, Characteristic Lyapunov exponents and smooth ergodic theory, Russian Math. Surveys 32 (1977), 55-114.

9. M. S. Raghunathan, A proof of Oseledec's multiplicative ergodic theorem, Israel J. Math. 32 (1979), 356-362.

10. D. Ruelle, Ergodic theory of differentiable dynamical systems, Inst. Hautes Études Sci. Publ. Math. 50 (1979), 27-58.

11. A. Ionescu Tulcea and C. Ionescu Tulcea, Topics in the theory of lifting, Ergeb. Math., Grenzgeb., Vol. 48, Springer-Verlag, Berlin and New York, 1969. 